
\title{
$\begin{array}{ll}\text { Research Square } & \begin{array}{l}\text { Preprints are preliminary reports that have not undergone peer review. } \\ \text { They should not be considered conclusive, used to inform clinical practice, } \\ \text { or referenced by the media as validated information. }\end{array}\end{array}$
}

\section{The Depression Anxiety Stress Scale 8: Its Psychometric Evaluation Among Women with Chronic Non-Cancer Pelvic Pain}

\author{
Amira Ali ( $\square$ mercy.ofheaven2000@gmail.com ) \\ Tokyo University of Agriculture and Technology \\ Amin Omar Hendawy \\ Tokyo University of Agriculture and Technology \\ Rasmieh Al-Amer \\ Isra University \\ Ghada Shahrour \\ Jordan University of Science and Technology \\ Esraa M. Ali \\ Alexandria University \\ Abdulmajeed A. Alkhamees \\ Qassim University \\ Nashwa Ibrahim \\ Mansoura University \\ Afaf Hassan Ahmed \\ Alexandria University \\ Sahar Mansour Taha Lamadah \\ Alexandria University
}

Research Article

Keywords: psychological distress/anxiety/depression, chronic pelvic pain, sexual abuse/assault, short form of the Depression Anxiety Stress Scale 21/Depression Anxiety

Posted Date: January 11th, 2022

DOI: https://doi.org/10.21203/rs.3.rs-1212124/v1

License: @ (i) This work is licensed under a Creative Commons Attribution 4.0 International License. Read Full License 


\section{Abstract}

Psychiatric comorbidity and abusive experiences in chronic pelvic pain (CPP) conditions may prolong disease course. This study investigated the psychometrics of the Depression Anxiety Stress Scale 8 (DASS-8) among women with CPP ( $N=214$, mean age $=33.3 \pm 12.4$ years). The DASS-8 expressed excellent fit, invariance across age groups and menopausal status, good discriminant validity (differentiating women with psychiatric comorbidity from those without comorbidity: $U=2018.0, p=0.001$ ), excellent reliability (alpha $=0.90$ ), adequate predictive and convergent validity indicated by strong correlation with the DASS-21 ( $r=0.94)$ and high values of item-total correlations ( $r=0.884$ to 0.893$)$. In two-step cluster analysis, it classified women into low and high distress clusters ( $\mathrm{n}=141$ and 73), with significantly higher levels of distress, pain severity and duration, and physical symptoms in cluster 2 . The DASS-8 correlated with pain severity/duration, depression/anxiety symptoms, sexual assault, fatigue, headache severity, and physical symptoms at the same level expressed by the parent scale, or even greater. Accordingly, distress may represent a target for early identification of psychiatric comorbidity, CPP severity, sexual assault, fatigue, etc. Therefore, the DASS-8 is a useful brief measure of mental symptoms among women with CPP.

\section{Introduction}

Pain in the pelvis unrelated to cancer, intercourse, or menstruation; which is experienced daily and persists for at least three consecutive months is known as chronic pelvic pain (CPP). CPP is commonly experienced by up to $22 \%$ of women [1-3]. It can be idiopathic or due to numerous urological, bowel-related, and gynecological reasons $[3,4]$. Because chronic noncancer pain conditions in women worsen over time, especially during the peak reproductive years,

fluctuations in ovarian hormones are suggested to be key modulators in the pathologies underlying CPP such as endometriosis and irritable bowel syndrome $[1,5,6]$.

Differences in CPP experience and pathology between reproductive age women and peri/postmenopausal women are reported in the literature [4]. Conditions entailing reduced levels of feminine hormones (e.g., menopause and during menses) are associated with increased sensitivity to visceral and pelvic pain [5]. In addition, ovarian collapse and subsequent reduction in estrogen during menopause is associated with numerous vegetative (e.g., hot flushes), physical (e.g., fatigue and back pain), urogenital/sexual (e.g., dyspareunia and urinary incontinence), cognitive (e.g., memory problems), and mental symptoms (e.g., depression and anxiety), which may aggravate pain sensitivity in midlife women and endanger their mental wellbeing [7]. In fact, CPP women within the age range of 25 to 35 years are reported to express less anxiety symptoms than their older counterparts [8].

The annual healthcare expenditure of CPP is enormous, exceeding 6.5 billion dollars in Australia [2]. In addition, CPP alters women's quality of life, reproductive capacity, social relations, work performance, and sexuality. As a result, CPP women experience high levels of distress, depression, and anxiety [9] Depression, anxiety, and mixed anxiety depression disorder (MADD) prevail in $63 \%, 66 \%$, and $54 \%$ of women experiencing CPP compared with $38 \%$, $49 \%$, and $28 \%$ of CPP-free women [8]. Exposure to different forms of abuse during early stages of life is associated with the development and persistence of numerous physical, emotional, mental, and sexual dysfunctions during adulthood [10]. Moreover, women in different parts of the world witness the highest exposure to different forms of abusive behavior against adults, along with numerous grave consequences [11]. Physical and sexual abuse represent a major risk factor for CPP.[3, 8] Given the traumatic origins of CPP and the distressful course of the condition, it is frequently managed within the biopsychosocial model of chronic pain-an approach that emphasizes the importance of wholistic management of chronic pain conditions "pain itself along with associated psychological and social problems" for more positive treatment outcomes [2]. However, CPP women experience varying levels of psychological distress. Greater comorbidity (e.g., depression, poor sleep, fatigue, somatic symptoms) is more common among women experiencing higher distress than those with little or no distress [12]. Therefore, careful identification of highly distressed CPP women through assessing psychopathological symptoms is extremely crucial for designing effective interventions for CPP and evaluating the outcomes of such interventions [2].

The Depression, Anxiety, and Stress Scale-21 (DASS-21) is a simple measure frequently used in research and clinical practice to capture the distinct features of depression, anxiety, and stress symptomatology [13]. However, numerous studies reported enormous flaws associated with the DASS-21: variations in its dimensional structure [14-17], invariance across different groups [18-20], and a ceiling effect [21]. As a result, many revisions of its item structure took place. Using several non-clinical samples, Osman et al. reported that the DASS-21 may be best used as a measure of psychological distress ${ }^{\text {[22] }}$-a state of emotional suffering that combines non-specific symptoms of depression and anxiety [23]. They also reported that numerous items poorly correlated with the underlying latent construct covered by the DASS-21, noting that reducing its items to 13 or nine items would remedy such flaws [22]. Subsequently, a 12-item version of the DASS-21 has been tested in non-clinical and clinical Korean samples [24]. However, this version has not been tested in another population until recently. A current study used clinical and non-clinical samples from Saudi Arabia to investigate various models of the parent scale as well as all the available shortened versions [25]. The fit of different models of the DASS-21 was considerably lower than all its short versions. An extensive revision of the item structure of the DASS-21 based on statistics and conceptual methods resulted in an 8-item version of the DASS-21. The DASS-8 expressed excellent psychometric properties compared with Osman's 13-item/nine-item DASS and the Korean DASS-12 [25]. Likewise, another investigation involving healthy individuals from the US, Australia, and Ghana, the DASS-8 expressed a better fit and more robust discriminant and criterion validity than the DASS-12. However, both the DASS-8 and DASS-12 exhibited variance at the scalar level across English-speaking and Ghanaian participants, with the latter expressing lower levels of distress [26]. Individuals' responses to items of the DASS-8 in different cultures may vary since individuals tend to selectively express their distress symptoms through culturally acceptable ways [27].

Brief screening instruments are intended to be used more frequently as clinical tools to facilitate the identification of pathological cases and enable assessing response to treatment. Accordingly, there is an intense need to ensure the relevance, local precision, and adequate sensitivity of these instrument [28]. To benefit from the DASS-8 as a brief measure of depression/anxiety and stress symptomatology, it needs to be evaluated in more diverse populations, including patient groups, to ensure the adequacy of its sensitivity. Therefore, this study aimed to examine the psychometrics of the DASS-8 in a sample of Australian women with CPP. We hypothesized that 1) the DASS-8 would express better fit and less variance than the DASS-12; 2) the DASS-8 would discriminate CPP women experiencing mental comorbidities from those without mental comorbidities; 3 ) the DASS- 8 would correlate with the DASS-21 at the same level 
expressed by the DASS-12; and 4) the DASS-8 would correlate with pain symptoms and history of sexual assault at the same level expressed by the parent scale.

\section{Material And Methods \\ Study design, participants, and procedure}

This study is a secondary analysis based on a publicly accessible dataset from a previously published cross-sectional study [3], which comprises a convenient sample of women with CPP. Data were collected via a pre-treatment self-administered questionnaire addressed to all clients attending a specialist pelvic pain clinic in South Australia over 18 months between January 2015 and July 2016. Women not signing informed consent, with several incomplete sections of the questionnaire, or solely experiencing period pain or dyspareunia were excluded from the study. Women or their guardian, if they were less than 18 years old, signed an informed consent. Because the protocol for data collection was previously approved by University of South Australia Human Research Ethics Committee (Application ID: 0000036598; 26/05/2017) [3], and the dataset was publicly accessible [29], we did not obtain an ethical approval for the current study.

\section{Measures}

The questionnaire used for data collection comprised a large set of questions about pain experienced, its intensity, duration, pain-free days per month, pain severity during sexual activity, severity of stabbing pelvic pain, pain severity on the day of data collection, experiencing (tiredness/fatigue, anxiety, low mood, bad headache), somatic symptoms (e.g., nausea, unusual sweating, dizziness, and bloating), history of sexual assault, current psychiatric disorders, etc. Questions addressing pain severity or intensity prompted the respondents to rate the intensity of pain on a scale from 0 (no pain) to 10 (extremely severe pain).

The Depression anxiety stress scale (DASS-21) was used to measure psychological distress. It comprises 21 items, in three subscales that assess symptoms of depression, anxiety and stress. Item responses are rated on a 4-point scale ranging from 0 (did not apply to me at all) to 3 (applied to me very much or most of the time). The minimum and maximum total scores of the DASS-21 are 0 and 63 [17, 30]. The DASS-8 comprises eight items, in three subscales: depression (three items e.g., felt down hearted and blue), anxiety (three items e.g., felt scared without reason), and stress (two items e.g., was using a lot of my mental energy) $[25,26]$. The total scores of the DASS- 8 and its subscales range between 0 to 24,0 to 9,0 to 9 , and 0 to 6 , respectively. The DASS- 12 comprises 12 items, in three subscales, each comprising four items. The total scores of the DASS-8 and its subscales range between 0 to 24 and 0 to 12, respectively [24]. The reliability of the DASS-21, DASS-8, and DASS- 12 in this sample is excellent (coefficient alpha $=0.95,0.90$, and 0.90 , respectively).

\section{Statistical analysis}

First, we checked the dataset for missing responses. Because multiple items of the DASS-21 had missing responses, we removed all participants with incomplete data on the DASS-8 and DASS-12, which resulted in a final sample size of 214 participants-response rate $=0.90 \%$. The normality of different version of the DASS were tested by Shapiro Wilk W test. The statistics of non-normal variables are reported as median (MD) and interquartile range (IQR; Q1Q3) while mean and standard deviation were used to report normally-distributed variables. Number and percentage were used to describe categorical variables.

Based on a former investigation [25], the factor structures of the DASS-8 and DASS-12 were examined using confirmatory factor analysis (CFA). To evaluate model fit as good or acceptable, we used chi square ( $\mathrm{X} 2$ ) index, ideally it should be non-significant, Comparative Fit Index (CFI) and Tucker-Lewis Index (TLI) equal to or above 0.95 and 0.90 , respectively, in addition to standardized root-mean-square residual (SRMR) and root mean square error of approximation (RMSEA) less than 0.06 and 0.08 , respectively $[7,31]$. This combination allows parsimonious evaluation of model fit because $\chi^{2}$ is largely dependent on sample size, which if used alone may disqualify well-fitting models that express minor misspecifications [30, 32]. Based on suggestions pointed by modification indices, few error terms were correlated to improve model fit.

To test measurement invariance of the DASS-8, age was categorized in two groups (156 below the age of 40 versus 58 aged 40 years and above). Then, multigroup CFA was conducted to compare invariance of the DASS-8 and DASS-12 at the configural, metric, scalar, and strict levels [33, 34] across groups of age, menopausal status (menopausal vs premenopausal), and psychiatric comorbidity (presence of comorbidity vs no comorbidity, the number of subjects in all the categories are shown in Table 1). Significant changes in $\chi^{2}$ in constrained models, along with $\triangle \mathrm{CFI}$ and $\triangle \mathrm{RMSEA}$ above 0.02 and 0.015 , respectively were used as criteria for non-invariance [23,33].

To examine the discriminant validity of the DASS-21, DASS-12, DASS-8, Mann Whitney U test was used to determine whether these measures and their subscales can differentiate CPP women with comorbid psychiatric disorders from those without psychopathology. It was also used to differentiate women with depressive disorder from those with anxiety disorder. In addition, heterotrait-monotrait (HTMT) ratio of correlations of items comprising the DASS-21, DASS-12, DASS-8 were computed [26, 35]. Moreover, two-step cluster analysis was used to determine whether the participants can be grouped according to the scores of the DASS-8 and its subscales. Two-step cluster analysis is a hybrid technique that operates via two steps. The first step (pre-clustering) employs a sequential approach to separate groups by pre-clustering cases based on a distance measure that defines dense regions in the analyzed attribute-space. In the second step (clustering), a probabilistic approach is used to statistically merge pre-clusters stepwise until the optimal subgroup model is determined. This technique is highly reliable; in terms of the number of subgroups detected, classification probability of individuals into subgroups, and reproducibility of the findings on different types of data. It has additional merits: analyzing atypical values (i.e., outliers), determining the number of clusters based on a statistical measure of fit rather than on an arbitrary choice, using categorical and continuous variables simultaneously, and handling large datasets [36]. Independent 
sample t-test, Mann Whitney test, and $\chi^{2}$ were used to compare the differences in mental symptomatology and characteristics of the participants across clusters.

The reliability of the DASS-21, DASS-12, DASS-8, and their subscales was assessed by coefficient alpha, alpha-if-item deleted, and item-total correlations. The predictive validity of the DASS-12, DASS-8, and their subscales was detected by Spearman's $r$ correlating these measures to the original scale and its subscales. This test was also used to evaluate the criterion validity of the three measures by correlating their scores with sexual assault experience, number of pain days/pain-free days per month, pain severity during sexual activity, pain severity on the day of data collection, severity of stabbing pelvic pain, experiencing bad headache, experiencing tiredness/fatigue, experiencing anxiety, and experiencing low mood. Additionally, a general structural equation path model (SEM) was constructed to use the DASS-8/DASS-12 and their subscales to predict these characteristics. Model fit was based on the same criteria used in CFA. Nonsignificant paths were trimmed to allow a better fit. All analyses were conducted in SPSS and Amos, and significance was considered at a probability level less than 0.05 in two-tailed tests.

\section{Results}

Characteristics of the participants

Participants in this study ( $\mathrm{N}=214$, mean age $=33.1 \pm 12.4$ years $)$ were women complaining from CPP. The participants experienced several types of pain (stabbing pelvic pain, sex pain, bowel pain, and headache), somatic symptoms (e.g., bloating, nausea, dizziness, unusual sweating), along with symptoms of low mood, anxiety, and psychiatric comorbidity. Table 1 shows more information on the sociodemographic and clinical characteristics of the participants in the overall sample as well as group differences in these variables between low distress and high distress women.

Results of confirmatory factor analysis and invariance analysis

As shown in Table 2, the one-factor structure of the DASS-8/DASS-12 expressed unsatisfactory fit. The crude models of the three-factor structures of the DASS-8 and the DASS-12 expressed good fit. Correlating the error terms of two items (Figure 1) in both models slightly improved the fit. The fit of the second order structure was similar to that of the three-factor structure of both scales (supplementary material). While the bifactor structure of the DASS-8 expressed good fit with all items significantly loading on the general factor, item 12 and item 20 failed to load on their domain-specific factors of stress and anxiety, respectively $(p>.05)$. In addition, item 15 loaded significantly on the anxiety factor $(p=.04)$, but its loading on this factor was weak $(\beta=.26)$. All items of the anxiety subscale of the DASS-12 failed to load on their corresponding factor in the bifactor model. Therefore, the three-factor structures of the DASS-8/DASS12 represent the best fit of the data.

As shown in Table 3, the three-factor structures of the DASS-8 and DASS-12 were invariant at the configural, metric, scalar, and strict levels across groups of age and menopausal status. However, the DASS-8 was non-invariant at the scalar level across groups of psychiatric comorbidity $(\triangle \mathrm{CFI}>0.02$ and $\triangle \mathrm{RMSEA}$ $>0.15)$. The DASS-12 was non-invariant at the strict level $(\triangle \mathrm{CFI}>0.02$ and $\triangle \mathrm{RMSEA}>0.15)$, and it also tended to be non-invariant at the scalar level across comorbidity groups $(\mathrm{SRMR}=0.1117)$.

Results of discriminant validity tests

As noted in Table 4, the DASS-21, DASS-8, DASS-12 differentiated between women with a current psychiatric disorder and those without at the same level of significance $(p<0.001)$. However, the $z$ scores of the DASS- 8 and its subscales were higher than those of the DASS-12 and the DASS- 21 , indicating a higher level of discriminant validity. Nonetheless, neither the DASS-21, DASS-8, DASS-12 nor their subscales could differentiate women with a diagnosis of depression from those with a diagnosis of anxiety. Likewise, the DASS-21, DASS-8, DASS-12, and their subscales significantly correlated with subjective experience of anxiety and low mood at the same levels (all p values $<0.01$, Table 4). The HTMT ratio of correlations show that the constructs covered by the subscales of the DASS-8 and DASS-12 were distinct $(<0.85)$. However, the stress and anxiety subscales on the DASS-8 were overlapping $($ HTMT ratio $=0.95)$. All the subscales of the parent scale exhibited overlap, albeit it was marginal for the stress-anxiety subscales (HTMT ratio $=0.85$ supplementary materials). 
Table 4

Descriptive statistics and discriminant validity of the Depression Anxiety Stress Scale 21 and its shortened versions among women with chronic pelvic pain

\begin{tabular}{|c|c|c|c|c|c|c|c|}
\hline \multirow[t]{2}{*}{ DASS versions } & $\begin{array}{l}\text { Whole sample } \\
(\mathrm{N}=214)\end{array}$ & Mann Whitney test & $\mathbf{z}$ & $\begin{array}{l}\text { Cluster } 1 \\
(n=141)\end{array}$ & $\begin{array}{l}\text { Cluster } 2 \\
(n=73)\end{array}$ & \multirow[t]{2}{*}{ Mann Whitney test } & \multirow[t]{2}{*}{$\mathbf{z}$} \\
\hline & MD (IQR) & & & MD (IQR) & MD (IQR) & & \\
\hline DASS-21 & $11.5(6.0-23.8)$ & 2247.5 & -5.49 & $7.0(4.0-11.8)$ & $29.5(23.0-36.8)$ & 210.0 & -11.43 \\
\hline Depression & $2.0(1.0-7.0)$ & 2495.5 & -5.08 & $1.0(0-3.0)$ & $9.0(5.0-14.0)$ & 1041.0 & -9.65 \\
\hline Anxiety & $3.0(1.0-7.0)$ & 2482.0 & -5.11 & $1.0(0-3.0)$ & $8.0(6.0-11.0)$ & 728.0 & -10.39 \\
\hline Stress & $6.0(3.0-11.0)$ & 2641.0 & -4.48 & $4.0(2.0-6.0)$ & $12.0(10.0-16.0)$ & 436.5 & -10.91 \\
\hline DASS-12 & $7.0(3.0-13.3)$ & 2320.0 & -5.48 & $4.0(2.0-7.0)$ & $17.0(13.0-21.0)$ & 397.0 & -11.08 \\
\hline Depression & $1.0(1.0-4.0)$ & 2266.5 & -5.80 & $0(0-1.0)$ & $5.0(3.0-8.5)$ & 1082.0 & -9.77 \\
\hline Anxiety & $2.0(0-4.0)$ & 2842.0 & -4.24 & $1.0(0-2.0)$ & $4.0(3.0-6.0)$ & 1514.5 & -8.63 \\
\hline Stress & $4.0(2.0-7.0)$ & 2849.5 & -4.16 & $2.0(1.0-4.0)$ & $8.0(6.0-9.5)$ & 856.0 & -10.05 \\
\hline DASS-8 & $4.0(1.0-8.0)$ & 2018.0 & -6.27 & $2.0(1.0-3.0)$ & $12.0(8.0-15.0)$ & 36.0 & -11.95 \\
\hline Depression & $1.0(0-3.0)$ & 2346.5 & -5.60 & $0(0-1.0)$ & $4.0(2.0-6.0)$ & 1214.0 & -9.47 \\
\hline Anxiety & $1.0(0-3.0)$ & 2521.0 & -5.21 & $0(0-1.0)$ & $4.0(3.0-6.0)$ & 674.0 & -10.93 \\
\hline Stress & $2.0(1.0-3.0)$ & 2447.0 & -5.26 & $1.0(0-2.0)$ & $4.0(3.0-5.0)$ & 364.0 & -11.37 \\
\hline
\end{tabular}

In two-step cluster analysis, the DASS-8 and its subscales classified the participants into two clusters: low distress (cluster 1: $\mathrm{n}=141,65.9 \%)$ and high distress (cluster $2: n=73,34.1 \%$ ). The model expressed good fit as indicated by Silhouette measure of cohesion and separation of around 0.7 and ratio of sizes less than 3 (1.93). Values of the predictor importance of the DASS-8 followed by stress, anxiety, and depression were $1,0.85,0.73$, and 0.5 , respectively. Mann Whitney $\mathrm{U}$ test revealed significant differences in the level of all mental distress symptoms among participants in both clusters-they were all significantly higher in cluster 2 than in cluster 1 (all p 0.001 ), with the DASS-8 and its stress subscale expressing the highest $z$ scores (Table 4).

Age, menopausal status, bloating, and the frequency of stabbing pelvic pain and sex pain did not vary significantly across clusters. However, participants in cluster 2 demonstrated significantly higher number of pain days $(t(146.9)=-2.50, p=0.014)$, less pain free days $(U=3654.0, z=-2.39, p=0.017)$; more severity of stabbing pelvic pain $(t(134.42)=-2.47, p=0.015)$, sex pain $(t(105.22)=-3.74, p=0.001)$, current pelvic pain $(U=3095.0, z=-3.29, p=0.001)$, concurrent headache $(t(118.11)=-3.51, p=0.001)$; higher frequency of bowel pain $\left(\chi^{2}(1)=6.68, p=.010\right)$, greater occurrence of sexual assault $\left(\chi^{2}(1)=19.06, p=.001\right)$, psychiatric co-morbidity $\left(\chi^{2}(2)=23.61, p=0.001\right)$, psychiatric symptoms (low mood $(\chi 2(16)=29.8, p=0.001)$ and anxiety $\left.\left(\chi^{2}(1)=50.50, p=0.001\right)\right)$, fatigue $\left(\chi^{2}(1)=14.12, p=0.001\right)$, sleep problems $\left(\chi^{2}(16)=12.13, p=0.001\right)$, and somatic symptoms (dizziness $\left(\chi^{2}(1)=7.57, p=0.006\right)$, unusual sweating $\left(\chi^{2}(1)=\right.$ $9.52, p=0.002)$, and nausea $\left.\left(\chi^{2}(1)=13.65, p=0.001\right)\right)$.

Results of tests of reliability, normality, and criterion validity

The reliability of the DASS-21, DASS-8, and DASS-12 was excellent. Meanwhile, the reliability of the shortened subscales ranged from very good to poor (Table 5)-poor reliability was reported only for the anxiety subscale of the DASS-12. The predictive validity of the DASS-8, DASS-12, and their subscales is depicted by their strong correlation with the original scale and its subscales (Table 5). The normality of the DASS-8 and the DASS-12 is comparable with that of the DASS-21 as noted by Shapiro-Wilks' W. As shown in Table 5, all the DASS versions and most of their subscales negatively correlated with pain-free days and positively correlated with pain experience on the survey day, pain days per month, concurrent headache, poor sleep, fatigue, somatic symptoms (nausea, bloating, dizziness, unusual sweating), experience of low mood and anxiety, as well as sexual assault experience. Notably, all the subscales of the DASS-8 correlated with sexual assault experience while the anxiety and stress subscales of the DASS-12 as well as the stress subscale of the DASS-21 could not correlate with this variable. Similarly, all subscales of the DASS-8 correlated with the severity of stabbing pelvic pain while the stress subscale of the DASS-12 and the DASS-21 failed to correlate with this variable. 
Table 5

Internal consistency, predictive validity, normality, and criterion validity of the Depression Anxiety Stress Scale (DASS) 21, DASS-12, DASS-8, and their subscale women with chronic pelvic pain

\begin{tabular}{|c|c|c|c|c|c|c|c|c|c|c|c|}
\hline Criteria & $\begin{array}{l}\text { DASS- } \\
21\end{array}$ & Depression & Anxiety & Stress & $\begin{array}{l}\text { DASS- } \\
12\end{array}$ & Depression & Anxiety & Stress & DASS-8 & Depression & Anxiety \\
\hline $\begin{array}{l}\text { Coefficient } \\
\text { alpha }\end{array}$ & 0.945 & 0.931 & 0.837 & 0.878 & 0.898 & 0.845 & 0.693 & 0.827 & 0.901 & 0.892 & 0.794 \\
\hline $\begin{array}{l}\text { Range of } \\
\text { corrected } \\
\text { item-total } \\
\text { correlations }\end{array}$ & $\begin{array}{l}0.401- \\
0.776\end{array}$ & $\begin{array}{l}0.598- \\
0.847\end{array}$ & $\begin{array}{l}0.370- \\
0.713\end{array}$ & $\begin{array}{l}0.593- \\
0.735\end{array}$ & $\begin{array}{l}0.401- \\
0.785\end{array}$ & $\begin{array}{l}0.607- \\
0.719\end{array}$ & $\begin{array}{l}0.371- \\
0.550\end{array}$ & $\begin{array}{l}0.607- \\
0.746\end{array}$ & $\begin{array}{l}0.629- \\
0.744\end{array}$ & $\begin{array}{l}0.882- \\
0.944\end{array}$ & $\begin{array}{l}0.634- \\
0.662\end{array}$ \\
\hline $\begin{array}{l}\text { Range of } \\
\text { alpha if- } \\
\text { item-deleted }\end{array}$ & $\begin{array}{l}0.940- \\
0.946\end{array}$ & $\begin{array}{l}0.914- \\
0.939\end{array}$ & $\begin{array}{l}0.795- \\
0.853\end{array}$ & $\begin{array}{l}0.851- \\
0.869\end{array}$ & $\begin{array}{l}0.881- \\
0.902\end{array}$ & $\begin{array}{l}0.787- \\
0.833\end{array}$ & $\begin{array}{l}0.585- \\
0.708\end{array}$ & $\begin{array}{l}0.742- \\
0.809\end{array}$ & $\begin{array}{l}0.884- \\
0.893\end{array}$ & $\begin{array}{l}0.831- \\
0.870\end{array}$ & $\begin{array}{l}0.702- \\
0.733\end{array}$ \\
\hline $\begin{array}{l}\text { Correlation } \\
\text { with } \\
\text { subscale of } \\
\text { the DASS- } \\
21\end{array}$ & - & - & - & - & - & $0.930^{\star \star \star}$ & $0.919^{* \star *}$ & $0.948^{\star \star \star}$ & - & $0.934^{\star \star \star}$ & $0.871^{\star \star \star}$ \\
\hline $\begin{array}{l}\text { Correlation } \\
\text { with the } \\
\text { DASS-21 }\end{array}$ & - & $0.856^{* \star *}$ & $0.823^{\star \star \star}$ & $0.929^{* \star *}$ & $0.966^{\star \star *}$ & $0.838^{* \star *}$ & $0.718^{* \star *}$ & $0.863^{* \star \star}$ & $0.943^{\star \star \star}$ & $0.811^{\star * *}$ & $0.789^{\star \star \star}$ \\
\hline $\begin{array}{l}\text { Shapiro } \\
\text { Wilk test }\end{array}$ & $0.892^{\star \star \star}$ & $0.812^{\star \star \star}$ & $0.848^{\star \star \star}$ & $0.936^{\star \star \star}$ & $0.906^{\star \star \star}$ & $0.787^{\star \star \star}$ & $0.843^{\star \star \star}$ & $0.933^{* \star \star}$ & $0.864^{\star \star \star}$ & $0.788^{\star \star \star}$ & $0.790^{\star \star \star}$ \\
\hline $\begin{array}{l}\text { Correlation } \\
\text { with sexual } \\
\text { assault } \\
\text { experience }\end{array}$ & $0.203^{\star \star}$ & $0.264^{\star \star \star}$ & $0.196^{\star *}$ & 0.134 & $0.192^{\star}$ & $0.278^{\star \star \star}$ & 0.095 & 0.106 & $0.259 * \star$ & $0.245^{\star \star}$ & $0.261^{\star \star \star}$ \\
\hline $\begin{array}{l}\text { Correlation } \\
\text { with pain } \\
\text { days/month }\end{array}$ & $0.183^{\star \star}$ & 0.137 & $0.221^{\star \star}$ & $0.183^{\star *}$ & $0.193^{\star \star}$ & $0.190 \star \star$ & $0.204^{\star \star}$ & 0.136 & $0.180^{\star}$ & $0.177^{\star}$ & $0.172^{\star}$ \\
\hline $\begin{array}{l}\text { Correlation } \\
\text { with pain- } \\
\text { free } \\
\text { days/month }\end{array}$ & $-0.182^{\star \star}$ & -0.132 & $-0.217^{\star \star}$ & $-0.174^{\star}$ & $-0.193^{\star \star}$ & $-0.188^{\star \star}$ & $0-.201^{\star *}$ & $-0.139 *$ & $-0.179 *$ & $-0.173^{\star}$ & $-0.168^{*}$ \\
\hline $\begin{array}{l}\text { Correlation } \\
\text { with } \\
\text { severity of } \\
\text { pain today }\end{array}$ & $0.265^{\star \star \star}$ & $0.217^{\star \star}$ & $0.331^{\star \star *}$ & $0.238^{\star \star}$ & 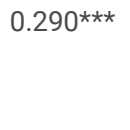 & $0.255^{\star \star \star}$ & $0.291^{\star * \star}$ & $0.239 * *$ & $0.265^{\star \star \star}$ & $0.225^{\star \star}$ & $0.274^{\star \star \star}$ \\
\hline $\begin{array}{l}\text { Correlation } \\
\text { with } \\
\text { severity of } \\
\text { stabbing } \\
\text { pelvic pain }\end{array}$ & $0.200 \star \star$ & $0.167^{\star}$ & $0.287 \star \star$ & 0.138 & $0.209 * \star$ & $0.174^{\star}$ & $0.246^{\star \star}$ & 0.143 & $0.205^{\star \star}$ & $0.149 *$ & 0.246 ** \\
\hline $\begin{array}{l}\text { Correlation } \\
\text { with } \\
\text { severity of } \\
\text { pain during } \\
\text { sexual } \\
\text { activity }\end{array}$ & $0.262^{\star \star}$ & $0.308^{\star \star \star}$ & $0.210^{\star}$ & $0.222^{\star \star}$ & 0.280 ** & 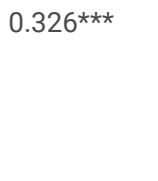 & $0.187^{\star}$ & $0.229 \star \star$ & 0.257 ** & $0.285^{\star \star}$ & $0.218^{\star \star}$ \\
\hline $\begin{array}{l}\text { Correlation } \\
\text { with } \\
\text { severity of } \\
\text { bad } \\
\text { headache }\end{array}$ & $0.387 \star \star \star *$ & 0.300 ** & $0.253^{\star \star}$ & $0.378^{\star \star \star}$ & $0.395^{\star \star \star}$ & 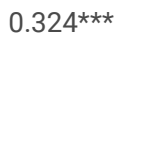 & $0.221^{*}$ & $0.363^{\star \star \star}$ & $0.299 * \star$ & $0.303^{\star \star}$ & $0.225^{\star}$ \\
\hline $\begin{array}{l}\text { Correlation } \\
\text { with } \\
\text { experienced } \\
\text { anxiety }\end{array}$ & $0.517 \star \star$ & $0.430 \star \star$ & $0.412^{\star \star}$ & $0.491^{\star \star}$ & $0.498^{\star \star}$ & $0.427^{\star \star}$ & $0.323^{\star \star}$ & $0.478^{\star \star}$ & 0.531 ** & $0.425^{\star \star}$ & $0.473^{\star \star}$ \\
\hline $\begin{array}{l}\text { Correlation } \\
\text { with } \\
\text { experienced } \\
\text { low mood }\end{array}$ & $0.487 * \star$ & $0.482^{\star \star}$ & $0.323^{\star \star}$ & $0.446^{\star \star}$ & $0.478^{\star \star}$ & $0.512^{\star \star}$ & $0.227^{\star \star}$ & $0.447^{\star \star}$ & $0.514^{\star \star}$ & $0.492^{\star \star}$ & $0.400 * \star$ \\
\hline $\begin{array}{l}\text { Correlation } \\
\text { with } \\
\text { experienced } \\
\text { fatigue }\end{array}$ & $0.345^{\star \star}$ & $0.296^{\star \star}$ & $0.333^{\star \star}$ & $0.305^{\star \star}$ & $0.348^{\star \star}$ & $0.300 * *$ & $0.346^{\star \star}$ & $0.288^{\star *}$ & $0.322^{\star \star}$ & $0.283^{\star \star}$ & $0.231^{\star \star}$ \\
\hline
\end{tabular}

$*_{,} * *, * *$ : correlation is significant at a level of $0.05,0.01,0.001$, respectively. 


\begin{tabular}{|c|c|c|c|c|c|c|c|c|c|c|c|}
\hline Criteria & $\begin{array}{l}\text { DASS- } \\
21\end{array}$ & Depression & Anxiety & Stress & $\begin{array}{l}\text { DASS- } \\
12\end{array}$ & Depression & Anxiety & Stress & DASS-8 & Depression & Anxiety \\
\hline $\begin{array}{l}\text { Correlation } \\
\text { with poor } \\
\text { sleep }\end{array}$ & $0.303^{\star \star}$ & $0.250 \star \star$ & $0.337^{\star \star}$ & $0.248^{\star \star}$ & $0.305^{\star \star}$ & $0.227^{\star \star}$ & $0.329 * *$ & $0.238^{\star \star}$ & $0.283^{\star \star}$ & $0.246^{\star \star}$ & $0.240^{\star \star}$ \\
\hline $\begin{array}{l}\text { Correlation } \\
\text { with } \\
\text { dizziness }\end{array}$ & $0.251^{* *}$ & $0.174^{\star \star}$ & $0.333^{\star *}$ & 0.191 ** & $0.243^{* *}$ & $0.192^{\star \star}$ & $0.335^{\star \star}$ & $0.150^{*}$ & $0.244^{\star \star}$ & $0.181^{* \star}$ & $0.262^{\star \star}$ \\
\hline $\begin{array}{l}\text { Correlation } \\
\text { with } \\
\text { unusual } \\
\text { sweating }\end{array}$ & $0.238^{\star \star}$ & $0.182^{\star \star}$ & $0.280^{\star *}$ & $0.215^{\star \star}$ & $0.239 * *$ & $0.174^{\star}$ & $0.306^{\star *}$ & $0.197 \star \star$ & $0.222^{\star \star}$ & $0.179 \star \star$ & $0.189^{\star \star}$ \\
\hline $\begin{array}{l}\text { Correlation } \\
\text { with nausea }\end{array}$ & $0.256^{\star \star}$ & $0.240^{\star \star}$ & $0.339 * \star$ & $0.180^{*}$ & $0.259 * *$ & $0.221^{\star *}$ & $0.349 * *$ & $0.145^{*}$ & $0.236^{* \star}$ & $0.219 * *$ & $0.260^{\star *}$ \\
\hline $\begin{array}{l}\text { Correlation } \\
\text { with } \\
\text { bloating }\end{array}$ & $0.259 * \star$ & 0.216 ** & $0.255^{\star \star}$ & $0.206^{* \star}$ & $0.247^{\star \star}$ & $0.213^{\star \star}$ & $0.234^{\star \star}$ & $0.193^{\star \star}$ & $0.279 \star \star$ & $0.245^{\star \star}$ & $0.234^{\star \star}$ \\
\hline
\end{tabular}

After trimming non-significant paths, SEM used to test criterion validity of the DASS-8/DASS-12 expressed good fit $(\chi 2(16,16)=10.677,6.956 ; p=0.829$, 0.974 ; both $\mathrm{CFI}=1.00$, both $\mathrm{TLI}=1.00$, both RMSEA $=0.00$, SRMR $=0.0420,0.0376$ ). Scores of the DASS-8/DASS- 12 significantly predicted current pain severity, poor sleep, and sex pain. Scores of their anxiety subscales predicted concurrent headache while scores of their stress subscales predicted the number of pain days per month (Figure 2). The DASS-8 and DASS-12 exerted significant indirect effects on headache through current pain severity ( $\beta=-0.055,95 \%$ Cl: -0.120 to $-0.011, p=0.023)$ and $(\beta=-0.067,95 \% \mathrm{Cl}:-0.142$ to $-0.018, p=0.011)$, respectively as well as on sex pain through poor sleep $(\beta=0.070,95 \% \mathrm{Cl}: 0.023$ to $0.114, p=0.011)$ and $(\beta=0.073,95 \% \mathrm{Cl}: 0.031$ to $0.118, p=0.011,0.007)$, respectively. Month pain days mediated the effect of the stress subscale of the DASS-8 and DASS-12 on sex pain ( $\beta=-0.045,95 \% \mathrm{Cl}:-0.091$ to $-0.007, p=0.037)$ and $(\beta=-0.042 ; 95 \%$ Cl: -0.100 to $-0.009 ; p=0.041)$, respectively.

\section{Discussion}

Distress symptoms are excessively experienced in CPP patients [2, 8, 9], and its management can considerably affect disease recovery [2]. Because not all CPP women express higher levels of distress and resources directed toward management of distress are limited [12], there is a great need for brief measures that may facilitate the identification of highly distressed patients as well as response to treatment [28]. Using numerous robust psychometric testing techniques, the present study reports usefulness of the DASS-8 as a measure of distress, depression, anxiety, and stress symptomatology among Australian women with CPP.

The crude models of the three-factor structure of the DASS-8/DASS-12 expressed better fit than all other crude models. RMSEA was on the high side for the DASS-8, suggesting minor misspecifications in item loadings. The fit of this model was considerably improved by correlating the residuals of item 12 "I found it difficult to relax" on the stress subscale and item 13 "I felt down-hearted and blue" on the depression subscale. Likewise, the fit of the DASS-12 was slightly improved by correlating the residuals of item 12 and item 1 "I found it difficult to wind down" on the stress subscale. Accordingly, the final model of the DASS8 expressed a perfect fit on all CFA fit indices, superior to the DASS-12, indicating a better construct validity of the DASS-8 as we hypothesized. Meanwhile, the fit of the second-order factor structure of both scales was similar to the three-factor structure (supplementary material), indicating usability of the total score and the score of the subscales of these scales.

The three-factor structures of both scales exhibited configural, metric, scalar, and strict invariance across groups of age and menopausal status. Across groups of psychiatric comorbidity, the DASS-8 was non-invariant at the scalar level and the DASS-12 expressed misfit (indicating tendency toward noninvariance) at the scalar level and it was non-invariant at the strict level. Critical ratios for differences between parameters noted that the source of noninvariance of the DASS-8 involved: 1) lower loadings of item 20 "I felt scared without any good reason" on the anxiety subscale and item 12 on the stress subscale, 2) weaker correlation between the anxiety and depression subscales, and 3) less covariance between items 12 and 13 . All were noted among participants not diagnosed with a mental disorder compared with those having a psychiatric disorder. Non-invariance of the DASS-12 involved differences in the loading of item 17 "I felt I was not worth much as a person" and the variances of items 1, 3 "I couldn't seem to experience any positive feeling at all", and 10 "I felt that I have nothing to look forward"-all on the depression subscale. Investigations of discriminant validity revealed significant differences in all distress scores between both groups.

The DASS-8 can be a beneficial brief measure for identifying CPP women with psychiatric comorbidity, high level of distress, and other debilitating symptoms (e.g., greater pain severity, poor sleep, concurrent headache, gastrointestinal discomfort, etc.). This is because the DASS-8 expressed adequate discriminant validity by differentiating CPP women with psychiatric comorbidities from those who are mental-illness free. Although the discriminant validity of the DASS-8, DASS-12, and DASS-21 was expressed at the same level of significance $(p=0.001)$, the $z$ scores associated with the DASS- 8 were higher than those of the DASS-12 and DASS-21 (Table 4), indicating superior discriminant validity of the DASS-8. This finding is consistent with a former investigation in which the DASS-8 potently identified psychiatric patients from healthy subjects [25]. On the other side, the DASS-8 as well as the DASS-12 and the DASS-21 could not discriminate CPP women with depression disorder from those with anxiety disorder. In line, the DASS-21 and the DASS-12 have been previously reported to lack the capacity to differentiate depression disorder from anxiety disorder [16, 24, 37]. These findings are consistent with other results on the reported comorbidity of both depression and anxiety disorders, confirming that the DASS measure is not a clinically diagnostic tool, but it can efficiently identify 
individuals prone to both depression and anxiety psychopathologies [38, 39]. This can be a merit of a brief self-administered measure. Extra diagnostic workout can be performed in a next step to attain a formal diagnosis.

In two-step cluster analysis, the DASS-8 and its subscales classified CPP women into two clusters with low and high levels of distress. The DASS-8 and its stress subscale had higher predictor importance values than anxiety and depression. Mann Whitney U test revealed significant differences (all $p$ values $=$ 0.001) in the scores of all the DASS measures among women in both clusters, with the DASS-8 and its stress subscale exhibiting the highest $z$ scores (Table 4). As shown in Table 1, participants in cluster 2 (high distress) scored significantly higher on symptoms of pain severity, bowel pain, depression, anxiety, fatigue, dizziness, nausea, and unusual sweating than participants in cluster 1. This finding is consistent with a former study, which classified women into two clusters: one cluster comprised women high on depression, fatigue, and poor sleep while the other cluster comprised women experiencing no or minimal levels of these symptoms [12]. However, Mann Whitney U test, cluster analysis, and SEM in the present study indicate stronger discriminant validity of the DASS-8 and its stress and anxiety subscales than the depression subscale. Overall, the DASS-8 can be efficiently used to differentiate highly distressed CPP women (e.g., with psychiatric comorbidity and more severity of mental symptoms, pain, and physical symptoms) from those with low levels of distress.

Apart from good fit, invariance, and adequate discriminant validity, the DASS-8 demonstrates other excellent psychometric characteristics. As shown in Table 5 , the internal consistency of the DASS-8 and all its subscale was good-considerably higher than that of the DASS-12 as we hypothesized, except for the stress subscale, which comprises half the number of items on the corresponding subscale of the DASS-12. Whereas the values of alpha-if-item deleted indicated reduction in the reliability of the DASS-8, they indicated an increase in the reliability of the DASS-12 and its anxiety subscale (Table 5), lending support to the more robust convergent validity of the DASS-8. Consistent with our preset hypothesis, strong correlations of the DASS-8 and its subscales with the DASS-21 and its subscales convey adequate item coverage, predictive validity, and convergent validity. Generally, these results highlight the high homogeneity, specificity, and sensitivity of the items comprising the DASS-8, which have been reported among Arabs and English-speaking participants [25, 26].

In parallel, the DASS-8 and all its subscales exhibited significant correlations with sexual assault, pain duration (pain days per months), pain-free days, pain intensity, somatic symptoms, fatigue, poor sleep, bad headache, etc. On the other hand, the stress and depression subscales of the DASS-21 failed to correlate with sexual assault and pain-free days, respectively. Likewise, the anxiety and depression subscales of the DASS-12 failed to correlate with sexual assault while the correlation of the overall DASS-12 with sexual assault was at a lower level than that expressed by the DASS-8 $(r=0.192, p=0.05$ vs $r=0.259, p=$ 0.01). These findings indicate better criterion validity of the DASS-8. They are also congruent with recent studies reporting significant correlations of the subscales of the DASS-21 with stabbing pelvic pain, migraine, intimate partner violence/domestic violence; as well as early childhood physical, sexual and emotional abuse among women $[3,10,11,40]$. Moreover, SEM revealed that the DASS-8/DASS-12 and their subscales, except for depression, can directly and indirectly predict pain severity, pain days, sex pain, concurrent headache, and poor sleep (Figure 2). Therefore, the DASS-8 can be reliably used as a valid criterion to predict different noxious symptoms and experiences in CPP.

This study is the first attempt to confirm the psychometric soundness of the DASS-8 as a measure of mental symptomatology among Australian women with CPP. Results obtained from different robust testing techniques in this study emphasize excellent psychometric properties of the DASS-8; in terms of fit, invariance, predictive validity, convergent validity, discriminant validity, criterion validity, and reliability. These finding are all in line with those reported in Arab psychiatric patients as well as in healthy respondents from Saudi Arabia, Australia, the US, and Ghana.[25, 26] Nonetheless, the study has many limitations that should be acknowledged. The generalizability of the finding is limited out of possible risk for selection bias: 1) the sample is collected from a single clinic, 2) a priori power analysis for determining the sample size is lacking, 3) data on the respondents who declined participation in the survey are lacking, 4) inclusion and exclusion criteria are not clearly defined, and 5) the results are based on a convenience sample, which may not represent all CPP women in other settings or countries. The cross-sectional design used precludes test-retest reliability testing and casts doubt on the soundness of relations predicted in SEM. Thus, future investigations may use a longitudinal design to examine the properties of the DASS-8 as well as causal direction of effects addressed in this study, along with mechanisms underlying links between psychological distress, pain, sleep problems, concurrent headache, etc.

\section{Conclusion}

Psychometric evaluation of the DASS-8 among CPP women by numerous robust techniques revealed its proper fit, invariance, high reliability, good convergent validity, adequate predictive validity, and good criterion validity. The results indicate usability of the DASS-8 as a brief, reliable, invariant measure of mental symptoms in reproductive age and menopausal women with CPP. The Identification of highly distressed women would encourage the use of relevant psychiatric intervention. Owing to its brevity, the DASS-8 can facilitate frequent monitoring of common mental symptomatology over the course of CPP treatment, supporting efforts directed toward improving recovery in CPP population.

\section{Declarations}

Ethics approval and consent to participate: The protocol of data collection was approved by University of South Australia Human Research Ethics Committee (Application ID: 0000036598; 26/05/2017). All participants or their guardians signed a written informed consent before data collection [3]. No ethical approval was obtained for the current study because the analysis is based on a publicly accessible dataset [29]. The present study was conducted according to the Declaration of Helsinki.

Consent for publication: Not applicable.

Availability of data and materials: The dataset [29] supporting the conclusions of this article is available in Zenodo repository, [https://zenodo.org/record/1307252\#.YckoVWhBw2w], and also the datasets used and/or analyzed during the current study are available from the corresponding author on reasonable request. 
Acknowledgements: The authors would like to thank the Deanship of Scientific Research, Qassim University, for funding the publication of this paper.

\section{References}

1. Hassan, S.; Muere, A.; Einstein, G. Ovarian hormones and chronic pain: A comprehensive review. PAIN® 2014, 155, 2448-2460, doi:https://doi.org/10.1016/j.pain.2014.08.027.

2. Brooks, T.; Sharp, R.; Evans, S.; Baranoff, J.; Esterman, A. Psychological Interventions for Women with Persistent Pelvic Pain: A Survey of Mental Health Clinicians. J Multidiscip Healthc 2021, 14, 1725-1740, doi:10.2147/jmdh.s313109.

3. Brooks, T.; Sharp, R.; Evans, S.; Baranoff, J.; Esterman, A. Predictors of Depression, Anxiety and Stress Indicators in a Cohort of Women with Chronic Pelvic Pain. J Pain Res 2020, 13, 527-536, doi:10.2147/jpr.s223177.

4. Bartl, T.; Wolf, F.; Dadak, C. Pelvic congestion syndrome (PCS) as a pathology of postmenopausal women: a case report with literature review. BMC Womens Health 2021, 21, 181, doi:10.1186/s12905-021-01323-3.

5. Heitkemper, M.M.; Chang, L. Do fluctuations in ovarian hormones affect gastrointestinal symptoms in women with irritable bowel syndrome? Gend Med 2009, 6, 152-167, doi:https://doi.org/10.1016/j.genm.2009.03.004.

6. Zhang, Y.; Cao, C.; Du, S.; Fan, L.; Zhang, D.; Wang, X.; He, M. Estrogen Regulates Endoplasmic Reticulum Stress-Mediated Apoptosis by ERK-p65 Pathway to Promote Endometrial Angiogenesis. Reprod Sci 2021, 28, 1216-1226, doi:10.1007/s43032-020-00414-0.

7. Ali, A.M.; Ahmed, A.H.; Smail, L. Psychological Climacteric Symptoms and Attitudes toward Menopause among Emirati Women. Int. J. Environ. Res. Public Health 2020, 17, 5028, doi:10.3390/ijerph17145028

8. Siqueira-Campos, V.M.E.; Da Luz, R.A.; de Deus, J.M.; Martinez, E.Z.; Conde, D.M. Anxiety and depression in women with and without chronic pelvic pain: prevalence and associated factors. J Pain Res 2019, 12, 1223-1233, doi:10.2147/.jpr.s195317.

9. Della Corte, L.; Di Filippo, C.; Gabrielli, O.; Reppuccia, S.; La Rosa, V.L.; Ragusa, R.; Fichera, M.; Commodari, E.; Bifulco, G.; Giampaolino, P. The Burden of Endometriosis on Women's Lifespan: A Narrative Overview on Quality of Life and Psychosocial Wellbeing. International Journal of Environmental Research and Public Health 2020, 17, 4683.

10. Dye, H.L. Is Emotional Abuse As Harmful as Physical and/or Sexual Abuse? Journal of Child \& Adolescent Trauma 2020, 13, 399-407, doi:10.1007/s40653-019-00292-y

11. Malik, M.; Munir, N.; Ghani, M.U.; Ahmad, N. Domestic violence and its relationship with depression, anxiety and quality of life: A hidden dilemma of Pakistani women. Pak J Med Sci 2021, 37, 191-194, doi:10.12669/pjms.37.1.2893.

12. Ayorinde, A.A.; Bhattacharya, S.; Druce, K.L.; Jones, G.T.; Macfarlane, G.J. Chronic pelvic pain in women of reproductive and post-reproductive age: a population-based study. Eur J Pain 2017, 21, 445-455, doi:10.1002/ejp.938.

13. Lovibond, P.F.; Lovibond, S.H. Manual for the Depression Anxiety Stress Scales (2nd ed.). Psychology Foundation, Sydney 1995.

14. Vaughan, R.S.; Edwards, E.J.; Maclntyre, T.E. Mental Health Measurement in a Post Covid-19 World: Psychometric Properties and Invariance of the DASS21 in Athletes and Non-athletes. Front Psychol 2020, 11, 590559, doi:10.3389/fpsyg.2020.590559.

15. Henry, J.D.; Crawford, J.R. The short-form version of the Depression Anxiety Stress Scales (DASS-21): construct validity and normative data in a large nonclinical sample. Br J Clin Psychol 2005, 44, 227-239, doi:10.1348/014466505×29657.

16. Yıldırım, A.; Boysan, M.; Kefeli, M.C. Psychometric properties of the Turkish version of the Depression Anxiety Stress Scale-21 (DASS-21). British Journal of Guidance \& Counselling 2018, 1-14, doi:10.1080/03069885.2018.1442558.

17. Ali, A.M.; Ahmed, A.; Sharaf, A.; Kawakami, N.; Abdeldayem, S.M.; Green, J. The Arabic Version of The Depression Anxiety Stress Scale-21: Cumulative scaling and discriminant-validation testing. Asian J Psychiatr 2017, 30, 56-58, doi:http://dx.doi.org/10.1016/j.ajp.2017.07.018.

18. Bibi, A.; Lin, M.; Zhang, X.C.; Margraf, J. Psychometric properties and measurement invariance of Depression, Anxiety and Stress Scales (DASS-21) across cultures. Int J Psychol 2020, doi:10.1002/ijop.12671.

19. Scholten, S.; Velten, J.; Bieda, A.; Zhang, X.C.; Margraf, J. Testing measurement invariance of the Depression, Anxiety, and Stress Scales (DASS-21) across four countries. Psychol Assess 2017, 29, 1376-1390, doi:10.1037/pas0000440.

20. Zanon, C.; Brenner, R.E.; Baptista, M.N.; Vogel, D.L.; Rubin, M.; Al-Darmaki, F.R.; Gonçalves, M.; Heath, P.J.; Liao, H.Y.; Mackenzie, C.S., et al. Examining the Dimensionality, Reliability, and Invariance of the Depression, Anxiety, and Stress Scale-21 (DASS-21) Across Eight Countries. Assessment 2020, 1073191119887449, doi:10.1177/1073191119887449.

21. Page, A.C.; Hooke, G.R.; Morrison, D.L. Psychometric properties of the Depression Anxiety Stress Scales (DASS) in depressed clinical samples. Br J Clin Psychol 2007, 46, 283-297, doi:10.1348/014466506x158996.

22. Osman, A.; Wong, J.L.; Bagge, C.L.; Freedenthal, S.; Gutierrez, P.M.; Lozano, G. The Depression Anxiety Stress Scales-21 (DASS-21): further examination of dimensions, scale reliability, and correlates. J Clin Psychol 2012, 68, 1322-1338, doi:10.1002/jclp.21908.

23. Ali, A.M.; Alkhamees, A.A.; Elhay, E.S.A.; Taha, S.M.; Hendawy, A.O. COVID-19-related psychological trauma and psychological distress among communitydwelling psychiatric patients: people struck by depression and sleep disorders endure the greatest burden Frontiers in Public Health 2022, doi:10.3389/fpubh.2021.799812.

24. Lee, E.H.; Moon, S.H.; Cho, M.S.; Park, E.S.; Kim, S.Y.; Han, J.S.; Cheio, J.H. The 21-Item and 12-Item Versions of the Depression Anxiety Stress Scales: Psychometric Evaluation in a Korean Population. Asian Nurs Res (Korean Soc Nurs Sci) 2019, 13, 30-37, doi:10.1016/j.anr.2018.11.006.

25. Ali, A.M.; Alkhamees, A.A.; Hori, H.; Kim, Y.; Kunugi, H. The Depression Anxiety Stress Scale 21: Development and Validation of the Depression Anxiety Stress Scale 8-item in Psychiatric Patients and the General Public for Easier Mental Health Measurement in a Post-COVID-19 World. Int. J. Environ. Res.

Page 9/11 
Public Health 2021, 18, 10142, doi:https://doi.org/10.3390/ijerph181910142.

26. Ali, A.M.; Hori, H.; Kim, Y.; Kunugi, H. The Depression Anxiety Stress Scale 8-items expresses robust psychometric properties as an ideal shorter version of the Depression Anxiety Stress Scale 21 among healthy respondents from three continents. Front Psychol 2022, accepted manuscript.

27. Office of the Surgeon General (US); Center for Mental Health Services (US); National Institute of Mental Health (US). Mental Health: Culture, R., and Ethnicity: A Supplement to Mental Health: A Report of the Surgeon General. Rockville (MD): Substance Abuse and Mental Health Services Administration (US);. Chapter 2 Culture Counts: The Influence of Culture and Society on Mental Health. 2001 Aug.

28. Sweetland, A.C.; Belkin, G.S.; Verdeli, H. Measuring depression and anxiety in sub-saharan Africa. Depress Anxiety 2014, 31, 223-232, doi:10.1002/da.22142.

29. Brooks, T. Predictors of depression, anxiety and stress indicators in a cohort of women with chronic pelvic pain data set. Zenodo 2018, doi:https://doi.org/10.5281/zenodo.1307252.

30. Ali, A.M.; Green, J. Factor structure of the depression anxiety stress Scale-21 (DASS-21): Unidimensionality of the Arabic version among Egyptian drug users. Subst Abuse Treat Prev Policy 2019, 14, 40, doi:https://doi.org/10.1186/s13011-019-0226-1.

31. Ali, A.M.; Hori, H.; Kim, Y.; Kunugi, H. Predictors of nutritional status, depression, internet addiction, Facebook addiction, and tobacco smoking among women with eating disorders in Spain. Frontiers in Psychiatry 2021, 12, 2001, doi:10.3389/fpsyt.2021.735109.

32. Ali, A.M.; Hendawy, A.O.; Ahmad, O.; Sabbah, H.A.; Smail, L.; Kunugi, H. The Arabic version of the Cohen perceived stress scale: factorial validity and measurement invariance. Brain Sciences 2021, 11, 419, doi:https://doi.org/10.3390/brainsci11040419.

33. Ali, A.M.; Hendawy, A.O.; Almarwani, A.M.; Alzahrani, N.; Ibrahim, N.; Alkhamees, A.A.; Kunugi, H. The Six-item Version of the Internet Addiction Test: Its development, psychometric properties, and measurement invariance among women with eating disorders and healthy school and university students. Int J Environ Res Public Health 2021, 18, 12341, doi:https://doi.org/10.3390/ijerph182312341.

34. Ali, A.M.; Hendawy, A.O.; Elhay, E.S.A.; Ali, E.M.; Alkhamees, A.A.; Kunugi, H.; Hassan, N.I. The Bergen Facebook Addiction Scale: Its psychometric properties and invariance among women with eating disorders. BMC Women's Health 2022, under review.

35. Henseler, J.; Ringle, C.M.; Sarstedt, M. A new criterion for assessing discriminant validity in variance-based structural equation modeling. Journal of the Academy of Marketing Science 2015, 43, 115-135, doi:10.1007/s11747-014-0403-8.

36. Benassi, M.; Garofalo, S.; Ambrosini, F.; Sant'Angelo, R.P.; Raggini, R.; De Paoli, G.; Ravani, C.; Giovagnoli, S.; Orsoni, M.; Piraccini, G. Using Two-Step Cluster Analysis and Latent Class Cluster Analysis to Classify the Cognitive Heterogeneity of Cross-Diagnostic Psychiatric Inpatients. Front Psycho/ 2020, 11, 1085, doi:10.3389/fpsyg.2020.01085.

37. Gloster, A.T.; Rhoades, H.M.; Novy, D.; Klotsche, J.; Senior, A.; Kunik, M.; Wilson, N.; Stanley, M.A. Psychometric Properties of the Depression Anxiety and Stress Scale-21 in Older Primary Care Patients. J Affect Disord 2008, 110, 248-259, doi:10.1016/j.jad.2008.01.023.

38. Lovibond, P.F.; Lovibond, S.H. The structure of negative emotional states: Comparison of the Depression Anxiety Stress Scales (DASS) with the Beck Depression and Anxiety Inventories. Behav Res Ther 1995, 33, 335-343, doi:https://doi.org/10.1016/0005-7967(94)00075-U.

39. Caetano, A.C.; Oliveira, D.; Gomes, Z.; Mesquita, E.; Rolanda, C. Psychometry and Pescatori projective test in coloproctological patients. Annals of gastroenterology 2017, 30, 433-437, doi:10.20524/aog.2017.0145.

40. Al-Hayani, M.; AboTaleb, H.; Bazi, A.; Alghamdi, B. Depression, anxiety and stress in Saudi migraine patients using DASS-21: local population-based crosssectional survey. Int J Neurosci 2021, 1-9, doi:10.1080/00207454.2021.1909011.

\section{Figures}

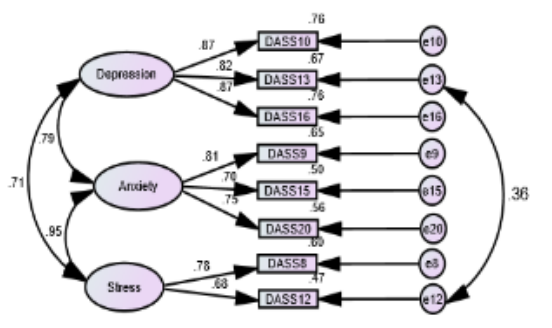

(a)

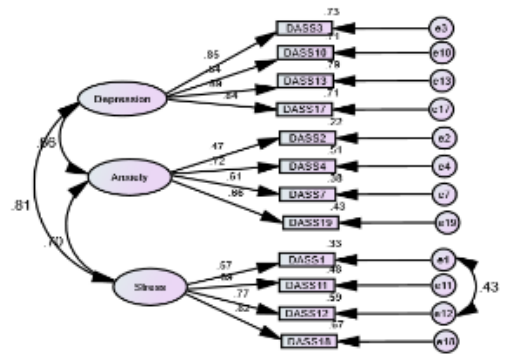

(b)

Figure 1

Factor structure of the short versions of the Depression Anxiety Stress Scale (DASS)-21: the DASS-8 (a) and the DASS-12 (b) among women with chronic pelvic pain. 

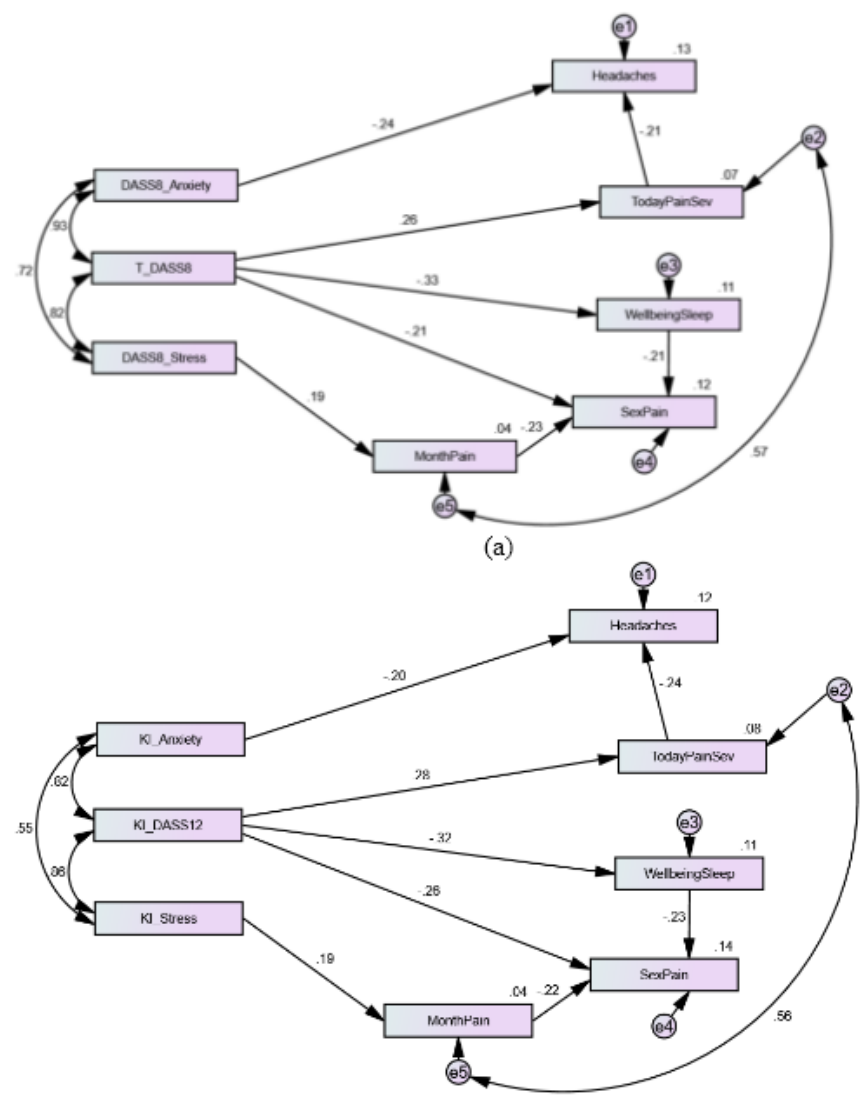

(b)

Figure 2

Structural equation model using the Depression Anxiety Stress Scale 8 (DASS-8) (a) and the DASS-12 (b) to predict pain intensity, pain days, sex pain, poor sleep, and concurrent headache among women with chronic pelvic pain.

\section{Supplementary Files}

This is a list of supplementary files associated with this preprint. Click to download.

- AppendixA..xlsx 